\title{
Epigenetic silencing of Wnt inhibition in systemic sclerosis
}

"Inhibition of DNA methylation might be an interesting novel approach to inhibit fibroblast activation by targeting canonical Wnt signalling," says Jörg Distler, as he summarizes the clinical implications of his new research in systemic sclerosis (SSc). By demonstrating that silencing of genes encoding the endogenous Wnt inhibitors Dickkopf-related protein 1 (also known as Dickkopf-1, DKK1) and secreted frizzledrelated protein 1 (SFRP1) by promoter hypermethylation contributes to aberrant Wnt signalling in SSc, the investigators raise the possibility that inhibitors of DNA methyltransferases (DNMTs) - already approved for use in other conditions - might be effective in patients with SSc.

\section{4 ...inhibitors of DNA methyltransferases....might be effective in patients with SSc 77}

"Tissue fibrosis caused by pathological activation of fibroblasts is a major hallmark of SSc," states Distler. Promoter hypermethylation (and consequent silencing) of anti-fibrotic genes is implicated in this process, he explains, as is increased Wnt signalling with decreased expression of endogenous inhibitors. To investigate a causal link between such changes, "we aimed to evaluate whether the expression of endogenous Wnt inhibitors might be silenced through DNA methylation," says Distler.

Methylation-specific PCR in samples from patients with SSc and from healthy controls (peripheral blood from 76 of each; lesional skin biopsies from 25 patients and 21 controls), revealed comparative promoter hypermethylation of DKK1 and SFRP1 in mononuclear cells and dermal fibroblasts from patients with SSc. Particularly in aSMA-positive myofibroblasts, immunohistochemistry showed subsequent decreased protein expression of these Wnt antagonists. "Of note," says Distler, "incubation of SSc fibroblasts with the DNMT inhibitor 5-aza-2'-deoxycytidine restored gene expression to control levels." Conversely, levels of $\beta$-catenin (central in canonical Wnt signalling) and expression of the Wnt target gene AXIN2 were substantially reduced in fibroblasts from patients with SSc by DNMT inhibition, which only minorly altered gene expression and $\beta$-catenin levels in healthy fibroblasts. The authors report similar results in mice with experimental fibrosis, in which DNMT inhibition also prevented bleomycininduced dermal thickening, accumulation of collagen and differentiation of resting fibroblasts into myofibroblasts.

Although well tolerated, DNMT inhibitors could prove to have unwanted effects in SSc, as pathways other than Wnt would be modulated. Adverse events aside, synergistic antifibrotic effects might boost the therapeutic potency of this approach.

Emma Leah

Original article Dees, C. et al. The Wnt antagonists DKK1 and SFRP1 are downregulated by promoter hypermethylation in systemic sclerosis. Ann. Rheum. Dis. doi:10.1136/annrheumdis-2012-203194 